\title{
TRIP13 Activates the PI3K/AKT Signal Pathway to Promote Melanoma Progression by Interacting With FLNA
}

\section{Wang Lu}

Zhongshan Hospital Fudan University

\section{Zhu Mengxuan}

Zhongshan Hospital Fudan University

\section{Zhang Yong}

Zhongshan Hospital Fudan University

\section{Ren Ming}

Zhongshan Hospital Fudan University

\section{Gao Zixu}

Zhongshan Hospital Fudan University

\section{Zhang Simin}

Zhongshan Hospital Fudan University

\section{Yang Yang}

Zhongshan Hospital Fudan University

\section{Qian Leqi}

Zhongshan Hospital Fudan University

\section{Shen Kangjie}

Zhongshan Hospital Fudan University

\section{Li Yanlin}

Zhongshan Hospital Fudan University

\section{Feng Jia}

Zhongshan Hospital Fudan University

\section{Ding Yiteng}

Zhongshan Hospital Fudan University

\section{Wei Chuanyuan}

Zhongshan Hospital Fudan University

Gu Jianying ( $\sim$ Prof_jianyinggu@163.com )

Zhongshan Hospital Fudan University 
Keywords: TRIP13, Melanoma, EMT, FLNA, PI3K/AKT

Posted Date: January 11th, 2022

DOI: https://doi.org/10.21203/rs.3.rs-1156586/v1

License: (c) (1) This work is licensed under a Creative Commons Attribution 4.0 International License. Read Full License 


\section{Abstract}

Background: Skin melanoma is a malignant tumor originated from skin melanocytes. It is highly malignant and easy to relapse and metastasis. Finding new diagnostic and therapeutic targets has become a hot issue. Accumulating evidence now indicates that thyroid hormone receptor interactor 13 (TRIP13) plays important roles in tumor development. However, its role in melanoma remains unclear.

Methods: Bioinformatic analyses were employed to excavate crucial genes in melanoma using several public databases. The expression of TRIP13 was detected by immunohistochemistry. MV3 cell and A2058 cell were steadily transfected with TRIP13 knock-down or overexpression lentiviruses, then the function and potential mechanism of TRIP13 were studied in vitro and in vivo. Co-immunoprecipitation (Co-IP) and mass spectrum were employed to screen out the interacting molecule of TRIP13.

Results: Our results showed that TRIP13 was generally upregulated in melanoma tissues and was related to the poor prognosis of melanoma patients. The overexpression of TRIP13 promotes the invasion, migration and EMT transformation of melanoma cells in vitro, and promotes lung metastasis in vivo. Mechanismly, TRIP13 interacts with FLNA, and activates the PI3K/AKT pathway, and then induces melanoma migration, invasion and EMT transformation.

Conclusion: Elevated TRIP13 drives tumor progression via the FLNA/PI3K/AKT axis, and TRIP13 is a innovative prognostic molecule and potential target of targeted therapy in melanoma.

\section{Introduction}

Melanoma is one of the fastest growing cutaneous malignant tumor, with an annual growth rate of $3 \%-5 \%[1]$. There are about 200000 new cases worldwide every year, resulting in about 55000 deaths $^{[2]}$. Somatic mutations occur repeatedly in all types of melanoma, for example, genes which regulate proliferation: BRAF, NRAS ${ }^{[3]}$; influence growth and metabolism PTEN and $\mathrm{KIT}^{[4]}$; resistance to apoptosis: TP53 ${ }^{[5]}$; participate in cell cycle control: CDKN2A $^{[6]}$. Therefore, targeted therapy for melanoma mutant genes came into being. For example, vemurafenib and dabrafenib were approved to treat metastatic and unresectable melanoma with BRAF mutation in 2011 and $2013^{[7,8]}$. However, many patients will develop drug resistance in a short time, which affects the efficacy of targeted drugs. Therefore, finding the pathogenesis and key molecules of melanoma will help researchers find new targeted therapies for melanoma.

ATPases Associated with Diverse Cellular Activity (AAA ATPase) is widely distributed in a variety of tissues and organs and affects a variety of life activities, such as DNA replication, protein degradation, membrane fusion, signal pathway transduction, regulation of gene expression and so on ${ }^{[9]}$. Studies have also found that it plays an important role in the occurrence and development of tumors ${ }^{[10]}$. RUVBL2, AAA ATPase, is necessary for the carcinogenic function of c-myb in AML by inhibiting myeloid differentiation ${ }^{[11]}$. ATAD3A (ATPase family AAA domain containing $3 A$ ) is affected by the up-regulation of 
miR-210-5P, which induces mitochondrial autophagy and sorafenib resistance in hepatoma cells ${ }^{[12]}$. However, there are few studies on AAA ATPase in melanoma. In 2021, Jilei Ma et al. utilised melanoma database: GSE3189 and GSE7553 to screen out the differentially expressed genes (DEGs) and established a protein-protein interaction network (PPI), and AAA ATPase family proteins-TRIP13 with a relatively high degree of connectivity were selected as one of the hub-genes that remarkably correlated to a poor survival rate ${ }^{[13]}$. However, the specific role of TRIP13 in the progression of melanoma has not been reported.

In this study, we explored the expression and clinical significance of TRIP13 in melanoma patients. Then we constructed TRIP13-Overexpression and TRIP13-knockdown cell line and further explore the biological function and underlying mechanism of TRIP13 both in in vivo and in vitro.

\section{Materials And Methods}

\section{Expression level of DEGs and survival analysis}

GSE3189, GSE7553 and GSE15605 were downloaded from the Gene Expression Omnibus (GEO) database (https://www.ncbi.nlm.nih.gov/geo). GEO2R (https://www.ncbi.nlm.nih.gov/geo/geo2r/) was used to calculate the adjusted p_values and logFC values among different groups. GEPIA(http://gepia.cancer-pku.cn/index.html), using the data of TCGA (cancer genome map) and GTEx (genotype tissue expression) can quickly provide useful information such as differential gene expression analysis between cancer and adjacent tissues and patient survival analysis ${ }^{[14]}$. The expression of TRIP13 in melanoma and the overall survival (OS) of patients was acquired from GEPIA.

\section{Patients and follow-up}

A total of 123 paraffifin-embedded melanoma and matched peritumor tissues (TMA) was used for immunohistochemistry $(\mathrm{IHC})$ analysis. All patients were received a complete excision then verified by a pathological examination. The patients did not receive radiotherapy and chemotherapy before operation. The clinical stage of patients was evaluated by the TNM staging system of the eighth edition of AJCC classification system. Ethical approval was obtained from the Ethics Committee of the Zhongshan Hospital Biomedical Research Department, and all patients signed informed consent.

\section{Immunohistochemistry (IHC)}

The tissue slides were deparaffinised in xylene and rehydrated in graded alcohol. After antigen retrieval by 1 mM EDTA buffer ( $\mathrm{pH} 8.0$ ), the slides then incubated in $0.3 \% \mathrm{H} 2 \mathrm{O} 2$ to block endogenous peroxidase activity. Subsequently, sections were incubated with goat serum, the TRIP13 antibody (sc-514314,Santa Cruz,1:50) / the FLNA antibody (sc-17749, Santa Cruz,1:50), Anti-mouse IgG HRP-linked Antibody (abcam,ab205719,1:2000), stained with DAB (Beyotime, Shanghai, China). The IHC grades were evaluated as previously described ${ }^{[15]}$. The score was determined based on a combination of staining percentage and intensity.The staining intensity was scored as follows: 0 (negative), 1 (low), 2 (moderate), or 3 (high), and the staining percentage was scored as follows: 1 (0-25\%), $2(>25-50 \%), 3(>50-75 \%)$ or 4 
(>75100\%). The sum was used for evaluating the expression level of TRIP13/FLNA and it was classified into two grades: low (03) or high(4-7).

\section{Cell culture and transfection}

Melanoma cell lines A2058 and MV3 were purchased from the cell bank of the Chinese Academy of Sciences (Shanghai, China), and were cultivated under the recommended conditions.Besides, A2058 expresses the BRAF (V600E), PTEN and p53 oncogenic mutations. The pSLenti-EF1a-EGFP-P2A-PuroCMV-MCS-3Flag and pRLenti-EF1a-EGFP-P2A- Puro-CMV-TRIP13-3Flag lentiviral vectors (Obio Technology, Shanghai, China) were transfected into A2058 cells. The shRNA targeting TRIP13 was synthesized and inserted into lentivirial vector GV493 (hU6-MCS-CBh-gcGFP-IRES-puro) from GeneChem, China. The shRNA1 sequence used was CCCATCGATTTGAGTGCAT and the shRNA2 sequence was GCTGAATTCCATGGGCTTT. Transfection efficiency was determined by Western blot. siRNAs against FLNA were designed and synthesized by Genomeditech (Shanghai, China). The target sequences were as follows: siFLNA, AGAAGAAGAUGCACCGCAA.

\section{Western blot and Immunofluorescence}

Western blot was completed as previously represented[16]. Melanoma cells were added with lysate to extract total protein, Suck $30 \mu$ g protein samples for $10 \%$ SDS -PAGE electrophoresis, transfer to PVDF membrane. Bands were blocked with $5 \%$ bovine serum albumin, and incubated with primary antibody as follows: anti-TRIP13 (sc-514314,Santa Cruz,1:1000), anti-GAPDH (5174, CST,1:1000), anti-AKT (4685,CST,1:1000), anti-p-AKT (4060S,CST,1:1000), anti-E-cadherin (sc-8426,SantaCruz,1:1000), anti-Ncadherin (ab18203, Abcam,1 $\mathrm{gg} / \mathrm{ml}$ ) and anti-FLNA (sc-17749,Santa Cruz,1:1000). The secondary antibodies used were as follows: Anti-rabbit IgG HRP-linked Antibody (7074,CST,1:1000) and Anti-mouse IgG HRP-linked Antibody (7076,CST,1:1000). Strip were added enhanced chemiluminescence solution and visualized by imaging system (Qinxiang, China).

For immunofluorescence staining, cells were fixed with $4 \%$ formaldehyde for $15 \mathrm{~min}$, infiltrated with $0.3 \%$ Triton X-100 (Beyotime, Shanghai, China) for 30 min at room temperature, and blocked with 5\% BSA. Cells were incubated with the primary antibodies overnight at $4^{\circ} \mathrm{C}$, washed by PBS for three times and then incubated by fluorescent secondary antibody. Then, cells were washed with PBS, stained with DAPI, and observed by Confocal fluorescence microscope (Olympus, Japan).

\section{Invasion and wound healing}

For CCK-8 assay, cells were inoculated into 96 -well plates (1000 cells/well). Then, cells were incubated with $10 \mu \mathrm{L}$ Cell Counting Kit-8 (Yeasen, Shanghai, China) for $2 \mathrm{~h}$ at $37^{\circ} \mathrm{C}$ after the $1 \mathrm{st}, 2 \mathrm{nd}$, 3rd and 4 th days. The absorbance values were measured at $450 \mathrm{~nm}$. For transwell invasion test, Matrigel (BD Biosciences, USA) was evenly spread into transwell chamber, and $10^{4}$ cells were spread into the transwell chamber with $200 \mu \mathrm{L}$ serum-free DEME; DMEM medium containing $20 \%$ FBS was added to the lower chamber. After $48 \mathrm{~h}$, take out the chamber, wash it with PBS and fix it in $4 \%$ paraformaldehyde solution for 
$30 \mathrm{~min}$; dyed with crystal violet for $15 \mathrm{~min}$ and take photos under the microscope. For wound healing assays, When the cells are full, use $200 \mu \mathrm{L}$ pipette tip scratch the bottom of the plate; Add serum-free DMEM medium and culture in $5 \% \mathrm{CO}_{2}$ cell incubator at $37^{\circ} \mathrm{C}$; The cells were photographed again at $48 \mathrm{~h}$, the migration rate was calculated by image $\mathrm{J}$.

In vivo assay

A2058-TRIP13 and A2058-Vector were suspended in PBS and a total of $10^{6}$ cells were injected subcutaneously into the left flank of mice. After 3 weeks, mice were sacrifificed, and the tumors were collected and weighed. For the metastasis model, $10^{6}$ cells were collected and were injected into the tail vein of nude mice. There were 6 mice in each group. The mice were killed 21 days after injection. The lungs were taken, fixed with $10 \%$ formaldehyde, sliced and stained with hematoxylin and eosin, photographed, and lung metastases were counted under the microscope.

\section{Co-immunoprecipitation (CO-IP) and MS}

Melanoma cells were collected by IP lysis solution and centrifuged. The primary antibody and Protein $\mathrm{A} / \mathrm{G}$ beads were added and mixed with the cell lysate at $4^{\circ} \mathrm{C}$ for $4 \mathrm{~h}$. Then,the precipitate was added into $40 \mu \mathrm{L}$ loading buffer for suspension. Regular sample loading, electrophoresis and luminescence. As for LC-MS/MS analysis, take an appropriate amount of peptide from each sample and use the nano liter flow rate easy NLC 1200 chromatographic system (Thermo Scientific) for chromatographic separation. Maxquant 1.6.1.0 mass spectrometry database retrieval software was used for retrieval.

\section{Statistical analysis}

The results are expressed as mean \pm standard deviation (SD). Student's $t$ tests was utilized to recognize the differences in two groups, one-way ANOVAs was utilized to recognize the differences among three or more groups. Kaplan-Meierand log-rank were utilized to analyze the overall survival and disease-free survival. A $p<0.05$ was considered statistically significant.

\section{Results}

\section{TRIP13 is highly expressed in melanoma and is associated with poor prognosis}

Three databases: GSE3189, GSE7553, GSE15605 were used to analyze differential genes between primary melanoma and normal skins. Among them, GSE3189 contains 7 normal skin and 45 primary melanoma; GSE7553 contained 4 normal skin and 14 primary melanoma; GSE15605 contained 16 normal samples and 46 primary melanoma. The expression profiles were analyzed by Limma package in $\mathrm{R}$, and P_value $<0.05$ and $\log _{2} \mathrm{FC}>1.3$ were used as thresholds. GSE3189 contained 7180 DEGs, 2841 upregulated genes and 4339 downregulated genes; GSE7553 contained 2857 DEGs, 1502 upregulated genes and 1355 downregulated genes; GSE15605 contained 8173 DEGs, 4428 upregulated genes and 3745 downregulated genes (Fig. 1A). We choose the upregulate genes in three databases(P_value $<0.05$ 
and $\log _{2} \mathrm{FC}>1.3$ ), and 65 DEGs overlapped in three databases (Fig. 1B). Then, overall survival analysis was performed based on 65 overlapped DEGs and the results showed that 24 DEGs are associated with poor prognosis of melanoma. Among them, TRIP13 has been reported to be involved in glioblastoma and chronic lymphocytic leukemia and overexpression TRIP13 signified poorer prognosis (Fig. 1D). ${ }^{[17,18] \text {; }}$ However, the expression level of TRIP13 in melanoma and its role in the progression of melanoma has not been studied.Therefore, we selected TRIP13 for further study. The level of TRIP13 mRNA in melanoma was tested with GEPIA. According to the database, the expression of TIRP13 raised in melanoma compared with normal skin (Fig. 1C). Then, we examined the expression of TRIP13 in our own cohorts of melanoma patients and revealed that TRIP13 was mainly in the cytoplasm (Fig. 1E). Through quantification analysis, We found that the content of TRIP13 in melanoma was higher than that in adjacent tissues (Fig. 1F). Importantly, patients in the TRIP13 ${ }^{\text {High }}$ group $(n=68)$ had poorer overall survival rates and disease-free survival than those in the TRIP13 ${ }^{\text {Low }}$ group $(n=55)(p=0.0042, p=0.0049$, Fig. 1G-H). In addition, high levels of TRIP13 were associated with lymph nodes metastasis $(p=0.002)$ and distant metastasis $(p=0.024)$ (Table 1$)$. Importantly, univariate and multivariate analyses showed that the TRIP13 levels were independent indicators of prognosis in patients with melanoma (Table 2). 
Table 1

Correlations Between TRIP13 with Clinicopathologic Features in 123 Melanoma Patients

\begin{tabular}{|c|c|c|c|}
\hline \multirow[t]{2}{*}{ Variable } & \multicolumn{2}{|c|}{ Number of patients } & \multirow[t]{2}{*}{ P value } \\
\hline & TRIP13 low & TRIP13 $3^{\text {high }}$ & \\
\hline \multicolumn{4}{|l|}{ Age,year } \\
\hline$<60$ & 25 & 24 & 0.397 \\
\hline$\geq 60$ & 32 & 42 & \\
\hline \multicolumn{4}{|l|}{ Gender } \\
\hline Male & 33 & 30 & 0.169 \\
\hline Female & 24 & 36 & \\
\hline \multicolumn{4}{|c|}{ Ulceration } \\
\hline Present & 45 & 58 & 0.181 \\
\hline Absent & 12 & 8 & \\
\hline \multicolumn{4}{|c|}{ Breslow depth (mm) } \\
\hline$\leq 2$ & 33 & 29 & 0.123 \\
\hline$>2$ & 24 & 37 & \\
\hline \multicolumn{4}{|c|}{ Clark level } \\
\hline I-III & 33 & 33 & 0.381 \\
\hline IV-V & 24 & 33 & \\
\hline \multicolumn{4}{|c|}{ Lymph nodes metastasis } \\
\hline No & 50 & 42 & 0.002 \\
\hline Yes & 7 & 24 & \\
\hline \multicolumn{4}{|c|}{ Distant metastasis } \\
\hline No & 46 & 41 & 0.024 \\
\hline Yes & 11 & 25 & \\
\hline \multicolumn{4}{|c|}{ Clinical stage } \\
\hline I-II & 33 & 42 & 0.515 \\
\hline III-IV & 24 & 24 & \\
\hline
\end{tabular}


Table 2

Univariate and Multivariate Analyses of Factors Associated With Overall Survival

\begin{tabular}{|c|c|c|c|c|}
\hline & \multicolumn{4}{|c|}{ Overall survival } \\
\hline & \multicolumn{4}{|c|}{ Multivariate Analysis } \\
\hline Variable & Univariate $\mathrm{P}$ & $\mathrm{HR}$ & $95 \% \mathrm{Cl}$ & $P^{*}$ \\
\hline Age, year ( $\geq 60$ vs. <60) & 0.166 & & & NA \\
\hline Gender (Men vs. Women) & 0.195 & & & NA \\
\hline Ulceration (Present vs. Absent) & 0.149 & & & NA \\
\hline Breslow depth (mm) ( $\leq 2$ vs. $>2$ ) & 0.543 & & & NA \\
\hline Clark level (I-III vs. IV-V) & 0.104 & & & NA \\
\hline Lymph nodes metastasis (Yes vs. No) & 0.143 & & & NA \\
\hline Distant metastasis (Yes vs. No) & 0.003 & 1.44 & $0.837-2.478$ & 0.188 \\
\hline Clinical stage (Yes vs. No) & 0.03 & 1.59 & $0.947-2.671$ & 0.079 \\
\hline TRIP13 staining (Low vs. High) & 0.003 & 1.933 & $1.171-3.188$ & 0.01 \\
\hline
\end{tabular}

TRIP13 promotes the invasion and migration of melanoma cells in vitro and in vivo

Then we constructed lentivirus-mediated stable expression of sh-TRIP13 in MV3 cells and cDNA vectormediated stable expression of TRIP13 in A2058 cells. The transfection efficiency was detected by Western blot (Fig. 2A). Knockdown of TRIP13 decreased the proliferation, migration and invasion of MV3 cells, and overexpression of TRIP13 increased the proliferation, migration and invasion of A2058 cells (Fig. 2B-D). Next, we investigated the effect of TRIP13 on tumorigenesis in vivo. However, there was no significant difference in tumor size between nude mice injected with A2058-Vector and nude mice injected with A2058-TRIP13 (Fig. 2E). In the pulmonary metastasis model, TRIP13-A2058 formed more lung metastases than Vector (Fig. 2F). Collectively, these results show that TRIP13 upregulation promotes melanoma progression both in vitro and in vivo.

\section{TRIP13 promotes EMT transformation of melanoma}

It was found that TRIP13 could promote the EMT transformation of tumor cells ${ }^{[19,20]}$. We detected the expression of TRIP13 and EMT marker in melanoma through GEPIA network, and found TRIP13 was positively correlated with $\mathrm{N}$-cadherin $(p=0.0043, r=0.13)$, Vimentin $(p=0.011, r=0.12)$,Snail $(p=0.00038$, $r=0.16)$, twist $(p=5.1 e-09, r=0.27)$, and ZEB2 ( $p=6.8 e-05, r=0.18)$ (Fig. 3A). We found that both MV3shRNAs and A2058-Vector cells showed epithelial characteristics, while both MV3-shNC and A2058- 
TRIP13 showed the characteristics of interstitial cells (Fig. 3B). By western blot and immunofluorescence analyses, we confirmed that high levels of TRIP13 increased the protein levels of N-cadherin, while low levels of TRIP13 increased protein levels of E-cadherin (Fig. 3C-D). These results show that elevated TPIP13 promotes the cellular EMT in melanoma.

\section{Overexpression TPIP13 lead to EMT by activating the PI3K/AKT pathway}

In order to explore the mechanism of TRIP13 induced EMT, we sequenced the cell lines overexpressing and knocking down TRIP13 (Fig. 4A). There were 399 upregulated DEGs and 415 downregulated DEGs in MV3-shNC cells compared with MV3-TRIP13 cells, while there were 1481 upregulated DEGs and 1640 downregulated DEGs in A2058-TRIP13 cells compared with A2058-Vector cells. Among them, 452 DEGs overlapped which KEGG analysis demonstrated were involved in pathways in cancer, focal adhesion, ECM-receptor interaction, regulation of actin cytoskeleton and PI3K/AKT signalling pathway (Fig. 4B).

According to the results of KEGG, we discovered that overexpression of TRIP13 enhanced the p-AKT, and that knock down of TRIP13 reduced p-AKT (Fig. 4C). Next, we found that TRIP13-induced migration, invasion were inhibited by LY-294002(AKT inhibitor, $50 \mu \mathrm{M}, 24 \mathrm{~h}$ ), an PI3K/AKT pathway inhibitor (Fig. 4D-E). Further, we found that TRIP13-induced upregulation of p-AKT and EMT markers were also inhibited by LY-294002 (Fig. 4F). Altogether, we verdict that TRIP13 accelerates EMT and tumour progression via PI3K/AKT signaling.

\section{TRIP13 binds to FLNA and activates the PI3K/AKT pathway}

To find the downstream protein of TRIP13, MS was conducted to find latent TRIP13-combining molecule in A2058-Vector cells and A2058-TRIP13 cells. Finally, 82 and 64 molecule were recognized in A2058Vector cells and A2058-TRIP13 cells, and twenty proteins only existed in A2058-TRIP13 cells (Fig. 5A). Among TRIP13 bound molecules, FLNA has been reported to be involved in the regulation of PI3K/AKT and $\mathrm{EMT}^{[21,22]}$. The interaction between TRIP13 and FLNA was confirmed by co-immunoprecipitation and immunofluorescence (Fig. 5B-C). Next, when FLNA was inhibited, the migration, invasion, EMT transformation and the activation of PI3K/AKT by upregulated TRIP13 also restrained (Fig. 5E-F). In sum, TRIP13 activates the PI3K/AKT pathway and induces EMT by interacting with FLNA.

\section{High expression of TRIP13 and FLNA indicated poor prognosis}

We then validated the relationship between TRIP13 and FLNA in our patient tissues, immunohistochemistry showed that TRIP13 was positively correlated with FLNA $(r=0.5949, p<0.0001$, Fig. 6A-B). Then, we examined the relationship between TRIP13 / FLNA and prognosis. it was found that patients with higher expression of TRIP13 / FLNA simultaneously had shorter overall survival and disease-free survival (Fig. 6C-D). This indicates that the high expression of TRIP13 and FLNA indicates bleak prognosis in patients with melanoma. Taken together, TRIP13 activates PI3K/AKT pathway, upregulates $\mathrm{N}$-cadherin and down-regulates E-cadherin by combining with FLNA, and then affects melanoma cell migration, invasion and EMT (Fig. 6E). 


\section{Discussion}

In this study, we explored the potential role and underlying mechanism of TRIP13 in melanoma. we demonstrates that TRIP13 is significantly upregulated in melanoma tissues, and is strongly correlated with poor prognosis in melanoma patients. Overexpression of TRIP13 is associated with a malignant phenotype of melanoma. Moreover, Our findings indicate that, TRIP13 combined with FLNA and activates the PI3K/AKT pathway and induces cell EMT.

A large number of literature reports that TRIP13 plays a key role in regulating mitotic processes, including spindle assembly checkpoint and DNA repair and result in chromosome instability $(\mathrm{CIN})^{[23,24]}$. Based on its own important physiological functions, abnormal expression of TRIP13 has been observed in various human cancers and related to several aspects of malignant phenotype, including excessive cell proliferation, drug resistance and tumor progression ${ }^{[25]}$. Liming Dong et al. found that TRIP13 regulates YWHAZ and promotes the proliferation, migration, invasion and EMT transformation of prostate cancer cells $^{[19]}$. TRIP13 promotes lung adenocarcinoma cell proliferation, clonogenicity, and migration and inhibiting apoptosis and G2/M phase shift as well in vitro ${ }^{[26]}$. In our experiment, we found that upregulation of TRIP13 promoted the invasion, migration, proliferation and EMT of A2058 cells via PI3K/AKT pathway. High levels of TRIP13 correlated with lymph nodes metastasis and distant metastasis. Importantly, univariate and multivariate analyses indicated that the TRIP13 level were independent indicators for melanoma patient prognoses.

AAA ATPase can facilitate the assembly or degradation of protein complexes ${ }^{[27]}$. TRIP13 has been reported to interact with functional proteins to activate related pathways and induce malignant transformation. In HCC, TRIP13 is regulated by miR-192-5p, interacted with ACTN4 and positively regulated its expression, thus activating the AKT/mTOR pathway to drive tumor progression ${ }^{[28]}$. In $\mathrm{CRC}$, TRIP13 interacted with a tyrosine kinase-FGFR4 and then activated the EGFR-AKT pathway and EMT ${ }^{[29]}$. In our experiment, we found that TRIP13 has a direct interaction with FLNA through CO-IP and mass spectrometry, and verified this interaction with Immunofluorescence. FLNA, the most abundant and widely distributed member of the filamin family (Filamin A, Filamin B and Filamin C), was first identified as a non-muscle actin filament crosslinking protein in $1975^{[30]}$. FLNA crosslinks actin filaments and helps to stabilize the cytoskeleton network and supports cell integrity ${ }^{[31]}$. Several studies showed that FLNA associates with multiple functional non-cytoskeletal proteins and is involved in several unrelated pathways that regulate cell migration and adhesion and promote tumor progression ${ }^{[32]}$. Mengmeng Cheng et al. demonstrated that c-Met-AKT-FLNA-smad formed a positive feedback loop to enhance EMT, which contributes to 5-FU resistance of CRC cells ${ }^{[33]}$. Consistently, our experiments showed that FLNA knockdown partly abolished TRIP13-induced EMT and activation of the PI3K/AKT pathway. Taking together, these results indicate that TRIP13 triggers PI3K/AKT signaling to promote EMT by interacting with FLNA. 
In conclusion, our findings confirmed the important role of TRIP13 in tumour progression and EMT via FLNA/PI3K/AKT pathway. We provides new insights into the mechanisms underlying melanoma progression. Our study offers novel evidence that TRIP13 may serve as a prognostic indicator and potential therapeutic target for melanoma patients.

\section{Abbreviations}

AAA ATPase: ATPase associated with a variety of cellular activities; CCK8: Cell counting kit-8; ECL: Electrogenerated chemiluminescence; EMT: Epithelial mesenchymal; GEPIA: Gene Expression Profiling Interactive Analysis; H\&E: Hematoxylin and eosin; HCC: Hepatocellular carcinoma; IHC: Immunohistochemistry; Co-immunoprecipitation: Co-IP; MS: Mass spectrometry; CRC: colorectal cancer; PBS: Phosphate buffer saline;PVDF: Polyvinylidene difluoride; ); FLNA: Filamin a; TMA: Tissue microarray; TRIP13: thyroid hormone receptor interactor 13; KEGG: Kyoto Encyclopedia of Genes and Genomes

\section{Declarations}

\section{Authors' contributions}

WCY, ZY and ZMX designed the study, WL, RM, GZX and DYT completed the experimental process, ZSM, LYL and YY conducted the statistical analyses, QLQ,SKJ and FJ wrote the paper, GJY revised the paper. All authors reviewed the manuscript. All authors read and approved the final manuscript.

\section{Acknowledgements}

We thank Airu Zhu at Shanghai Bioprofile Technology Company Ltd. for her technical support in mass spectroscopy.

\section{Funding}

This study was funded by the National Natural Science Foundation of China (81972559) and the Shanghai Shenkang Hospital Development Center Project (project no. SHDC2020CR2067B).

\section{Competing interests}

The authors declare that they have no competing interests.

\section{Consent for publication}

Not applicable.

\section{Ethics approval and consent to participate}

The Ethics Committee of the Zhongshan Hospital Biomedical Research Department provided ethical approval, and informed consent for collecting and preserving samples and details was obtained from 
each patient. Xenograft experiments in nude nice were approved by the Animal Experimentation Ethics Committee of Zhongshan Hospital, Fudan University.

\section{References}

1. TRIPP M K, WATSON M, BALK S J, et al. State of the science on prevention and screening to reduce melanoma incidence and mortality: The time is now[J]. CA Cancer J Clin, 2016,66(6): 460-480.

2. SCHADENDORF D, van AKKOOI A, BERKING C, et al. Melanoma[J]. Lancet, 2018,392(10151): $971-$ 984.

3. DELYON J, LEBBE C, DUMAZ N. Targeted therapies in melanoma beyond BRAF: targeting NRASmutated and KIT-mutated melanoma[J]. Curr Opin Oncol, 2020,32(2): 79-84.

4. KAPPELMANN-FENZL M, GEBHARD C, MATTHIES A O, et al. C-Jun drives melanoma progression in PTEN wild type melanoma cells[J]. Cell Death Dis, 2019,10(8): 584.

5. SCOLYER R A, LONG G V, THOMPSON J F. Evolving concepts in melanoma classification and their relevance to multidisciplinary melanoma patient care[J]. Mol Oncol, 2011,5(2): 124-136.

6. ZENG H, JORAPUR A, SHAIN A H, et al. Bi-allelic Loss of CDKN2A Initiates Melanoma Invasion via BRN2 Activation[J]. Cancer Cell, 2018,34(1): 56-68.

7. REBECCA $\vee$ W, SONDAK V K, SMALLEY K S. A brief history of melanoma: from mummies to mutations[J]. Melanoma Res, 2012,22(2): 114-122.

8. CHAPMAN P B, HAUSCHILD A, ROBERT C, et al. Improved survival with vemurafenib in melanoma with BRAF V600E mutation[J]. N Engl J Med, 2011,364(26): 2507-2516.

9. ZHANG G, LI S, CHENG K W, et al. AAA ATPases as therapeutic targets: Structure, functions, and small-molecule inhibitors[J]. Eur J Med Chem, 2021,219: 113446.

10. HURYN D M, KORNFILT D, WIPF P. p97: An Emerging Target for Cancer, Neurodegenerative Diseases, and Viral Infections[J]. J Med Chem, 2020,63(5): 1892-1907.

11. ARMENTEROS-MONTERROSO E, ZHAO L, GASPAROLI L, et al. The AAA+ATPase RUVBL2 is essential for the oncogenic function of c-MYB in acute myeloid leukemia[J]. Leukemia, 2019,33(12): 28172829.

12. WU H, WANG T, LIU Y, et al. Mitophagy promotes sorafenib resistance through hypoxia-inducible ATAD3A dependent Axis[J]. J Exp Clin Cancer Res, 2020,39(1): 274.

13. MA J, CAI X, KANG L, et al. Identification of novel biomarkers and candidate small-molecule drugs in cutaneous melanoma by comprehensive gene microarrays analysis[J]. J Cancer, 2021,12(5): 13071317.

14. TANG Z, LI C, KANG B, et al. GEPIA: a web server for cancer and normal gene expression profiling and interactive analyses[J]. Nucleic Acids Res, 2017,45(W1): W98-W102.

15. WEI C Y, ZHU M X, LU N H, et al. Bioinformatics-based analysis reveals elevated MFSD12 as a key promoter of cell proliferation and a potential therapeutic target in melanoma[J]. Oncogene, 2019,38(11): 1876-1891. 
16. WANG Q, QIAN L, TAO M, et al. Knockdown of DEAD-box RNA helicase 52 (DDX52) suppresses the proliferation of melanoma cells in vitro and of nude mouse xenografts by targeting c-Myc[J]. Bioengineered, 2021,12(1): 3539-3549.

17. ZHANG G, ZHU Q, FU G, et al. TRIP13 promotes the cell proliferation, migration and invasion of glioblastoma through the FBXW7/c-MYC axis[J]. Br J Cancer, 2019,121(12): 1069-1078.

18. ZHOU K, ZHANG W, ZHANG Q, et al. Loss of thyroid hormone receptor interactor 13 inhibits cell proliferation and survival in human chronic lymphocytic leukemia[J]. Oncotarget, 2017,8(15): 2546925481.

19. DONG L, DING H, LI Y, et al. TRIP13 is a predictor for poor prognosis and regulates cell proliferation, migration and invasion in prostate cancer[J]. Int J Biol Macromol, 2019,121: 200-206.

20. SHENG N, YAN L, WU K, et al. TRIP13 promotes tumor growth and is associated with poor prognosis in colorectal cancer[J]. Cell Death Dis, 2018,9(3): 402.

21. SHENG F, CHEN K X, LIU J, et al. Chromium (VI) promotes EMT by regulating FLNA in BLCA[J]. Environ Toxicol, 2021,36(8): 1694-1701.

22. KE J, WU G, ZHANG J, et al. Melanoma migration is promoted by prion protein via Akt-hsp27 signaling axis[J]. Biochem Biophys Res Commun, 2020,523(2): 375-381.

23. MARKS D H, THOMAS R, CHIN Y, et al. Mad2 Overexpression Uncovers a Critical Role for TRIP13 in Mitotic Exit[J]. Cell Rep, 2017,19(9): 1832-1845.

24. CLAIRMONT C S, SARANGI P, PONNIENSELVAN K, et al. TRIP13 regulates DNA repair pathway choice through REV7 conformational change[J]. Nat Cell Biol, 2020,22(1): 87-96.

25. LU S, QIAN J, GUO M, et al. Insights into a Crucial Role of TRIP13 in Human Cancer[J]. Comput Struct Biotechnol J, 2019,17: 854-861.

26. LI W, ZHANG G, LIX, et al. Thyroid hormone receptor interactor 13 (TRIP13) overexpression associated with tumor progression and poor prognosis in lung adenocarcinoma[J]. Biochem Biophys Res Commun, 2018,499(3): 416-424.

27. ZHANG S, MAO Y. AAA+ ATPases in Protein Degradation: Structures, Functions and Mechanisms[J]. Biomolecules, 2020,10(4).

28. ZHU M X, WEI C Y, ZHANG P F, et al. Elevated TRIP13 drives the AKT/mTOR pathway to induce the progression of hepatocellular carcinoma via interacting with ACTN4[J]. J Exp Clin Cancer Res, 2019,38(1): 409.

29. AGARWAL S, BEHRING M, KIM H G, et al. TRIP13 promotes metastasis of colorectal cancer regardless of p53 and microsatellite instability status[J]. Mol Oncol, 2020,14(12): 3007-3029.

30. HARTWIG J H, STOSSEL T P. Isolation and properties of actin, myosin, and a new actinbinding protein in rabbit alveolar macrophages[J]. J Biol Chem, 1975,250(14): 5696-5705.

31. KUMAR A, SHUTOVA M S, TANAKA K, et al. Filamin A mediates isotropic distribution of applied force across the actin network[J]. J Cell Biol, 2019,218(8): 2481-2491. 
32. WELTER H, HERRMANN C, FRÖHLICH T, et al. Filamin A Orchestrates Cytoskeletal Structure, Cell Migration and Stem Cell Characteristics in Human Seminoma TCam-2 Cells[J]. Cells, 2020,9(12).

33. CHENG M, JIANG Y, YANG H, et al. FLNA promotes chemoresistance of colorectal cancer through inducing epithelial-mesenchymal transition and smad2 signaling pathway[J]. Am J Cancer Res, 2020,10(2): 403-423.

Figures 
A

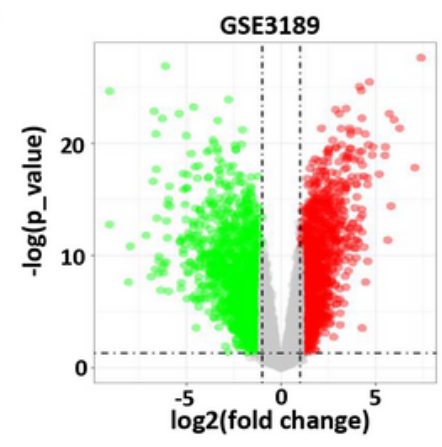

B

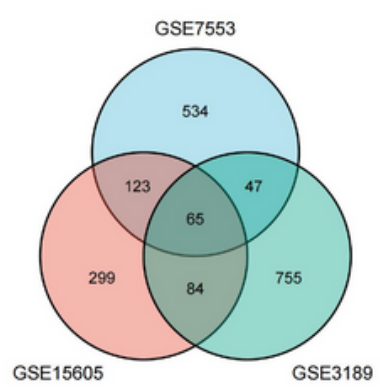

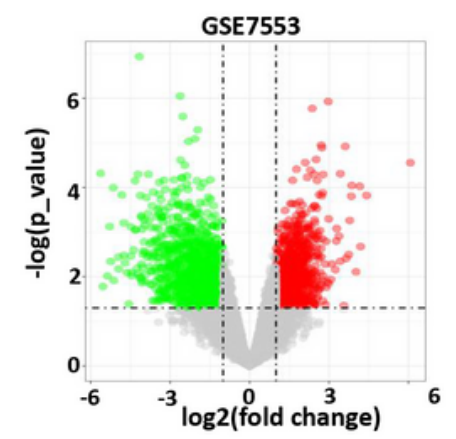

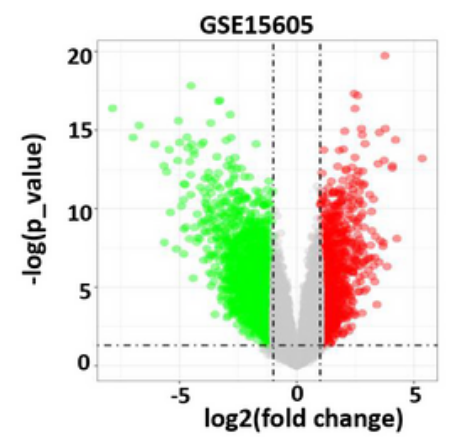

C

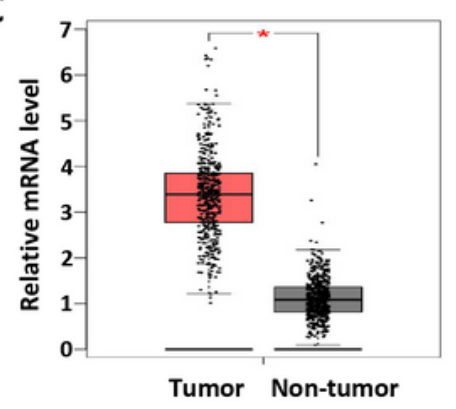

D

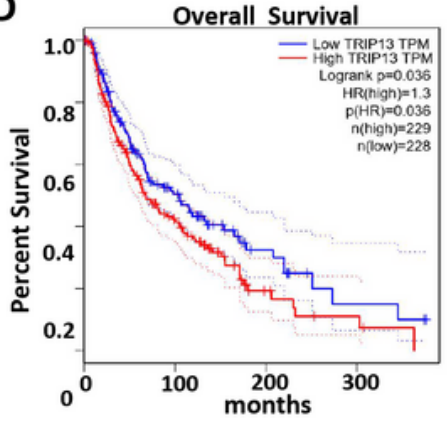

$\mathbf{E}$

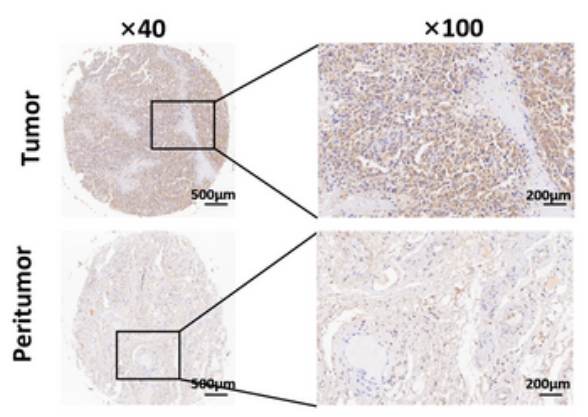

G

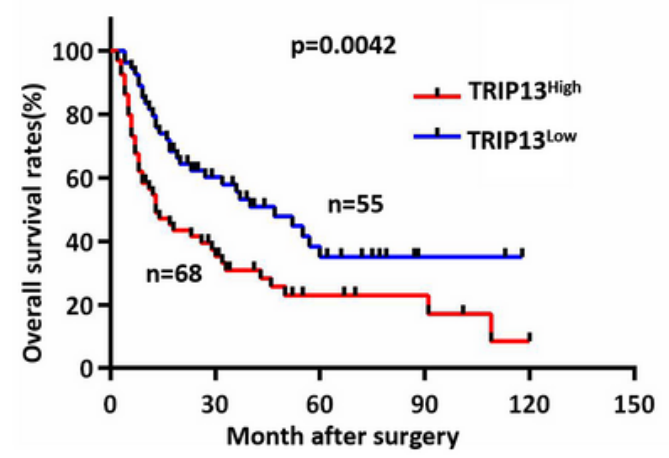

$\mathbf{F}$

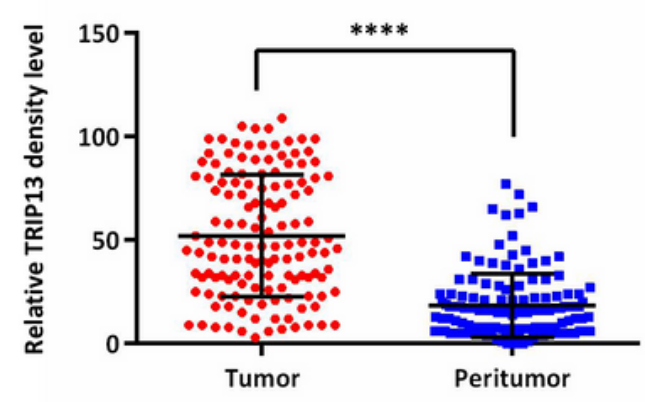

H

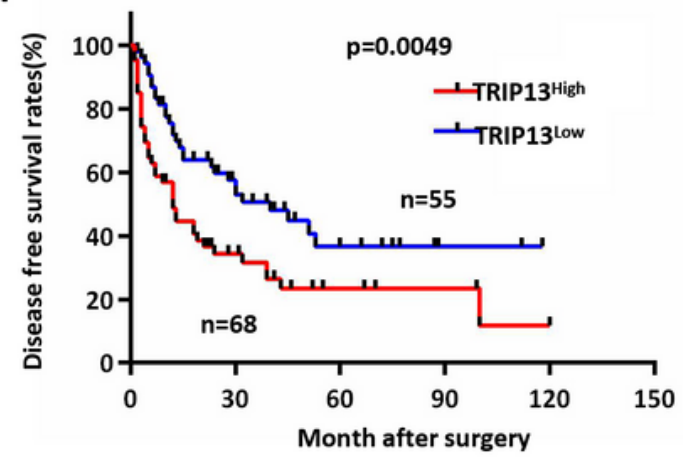

Figure 1

TRIP13 is highly expressed in melanoma and is associated with poor prognosis

A. Volcano plot of the differentially expressed genes in GSE3189, GSE7553 and GSE15605. B. 65 DEGs(logFC>1.3, p_value<0.05) overlapped in GSE3189, GSE7553 and GSE15605. C. TRIP13 mRNA expression in melanoma and normal tissue from GEPIA. D. Survival analysis of TRIP13 expression in 
melanoma patients from GEPIA. E-F. TRIP13 was tested by IHC in a TMA containing 138 melanoma patients. G-H. Overall survival and disease free survival analyses of 123 melanoma patients according to TRIP13 expression. ${ }^{* \star \star *} p<0.0001$

A

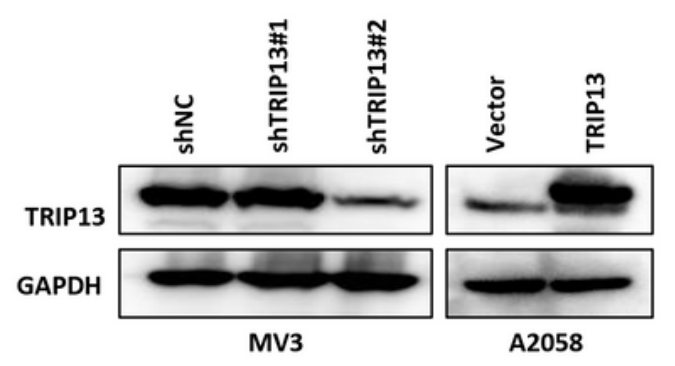

B

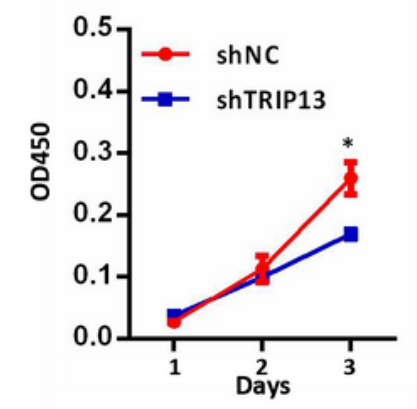

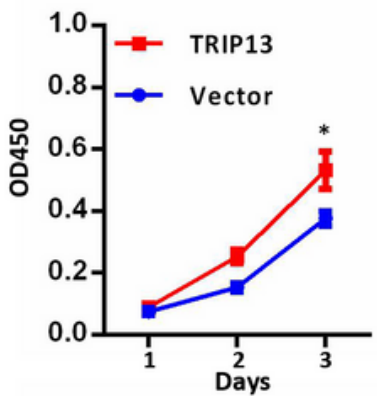

C
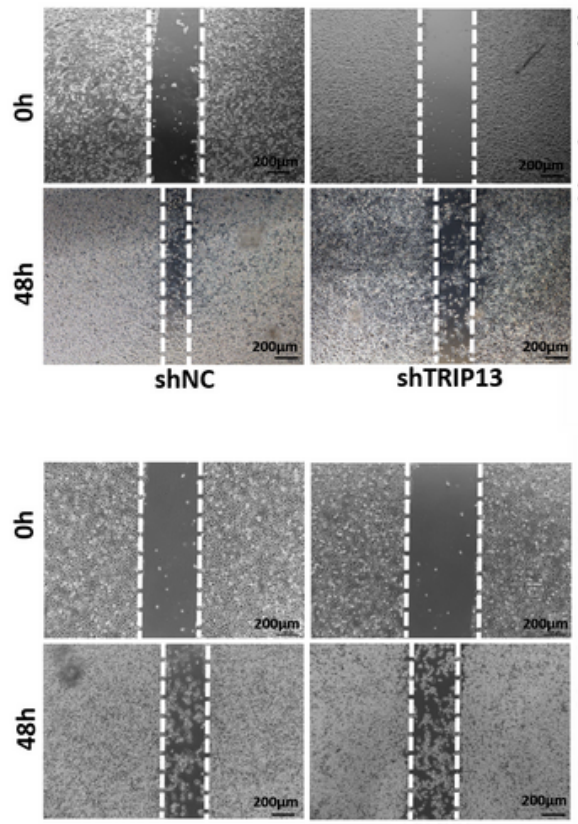

Vector
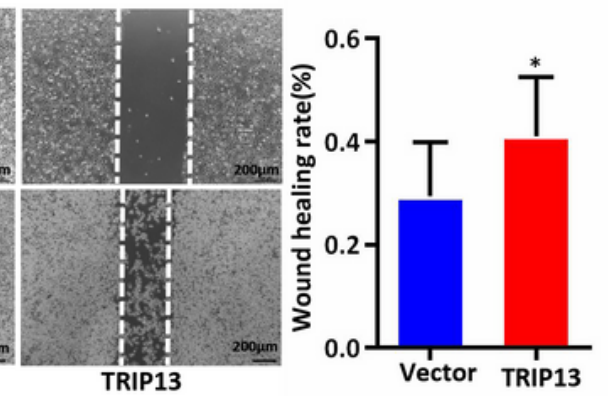

D
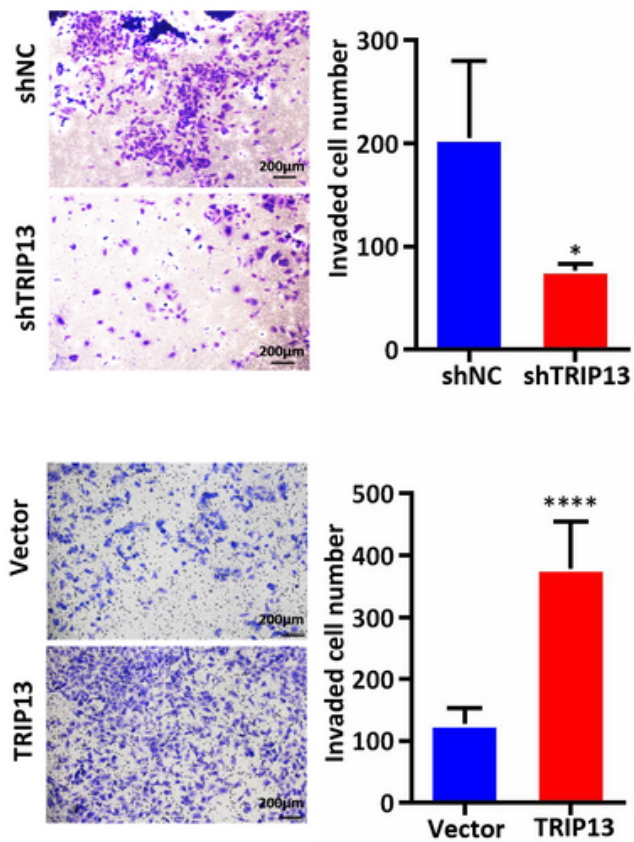

E

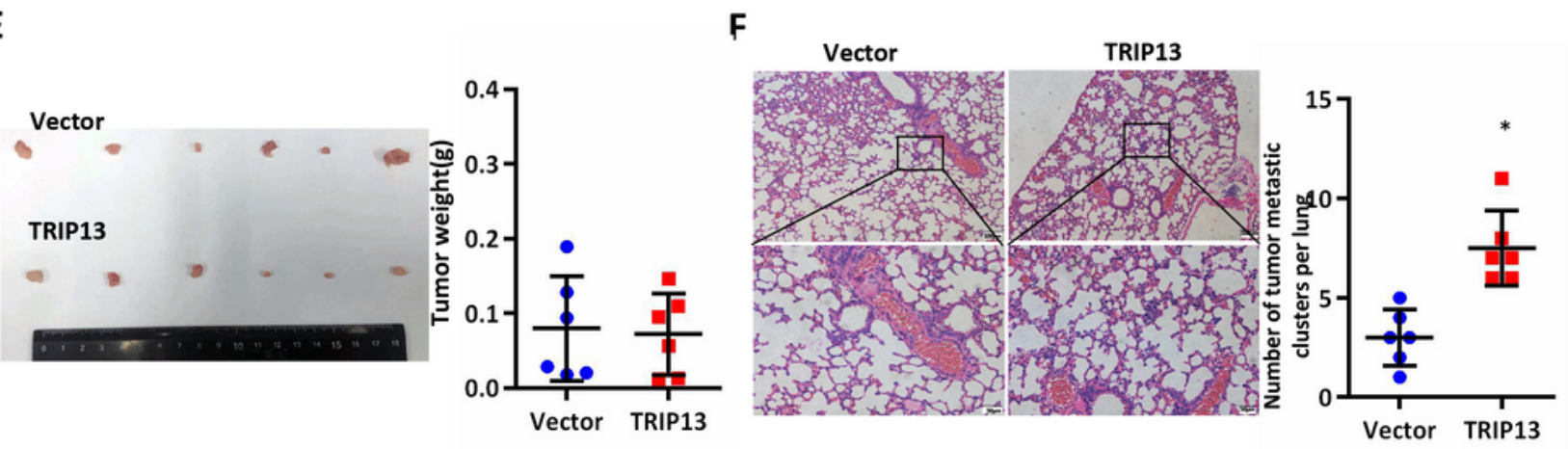

Figure 2 
TRIP13 promotes the invasion and migration of melanoma cells in vitro and in vivo A. The efficiency of transfection were detected by western blot. B. CCK-8 was used to detect the proliferation of overexpressed / knockdown TRIP13 melanoma. C. The migration ability of overexpressed / knockdown TRIP13 melanoma was detected by Wound-healing assays. D. The Invasive abilities of overexpressed / knockdown TRIP13 melanoma was detected by transwell assays. E. Tumors in nude mice bearing A2058shNC and A2058-TRIP13 cells and tumor weight were examined after 3 weeks. F. $10^{6}$ A2058-shNC and A2058-TRIP13 cells were injected into the tail vein of nude mice $(n=6)$. After 21 days, the lungs were obtained and Lung metastases were counted. ${ }^{\star} p<0.05$, ${ }^{\star \star} p<0.01,{ }^{\star \star \star} p<0.001$, ${ }^{\star \star \star \star} p<0.0001$

A
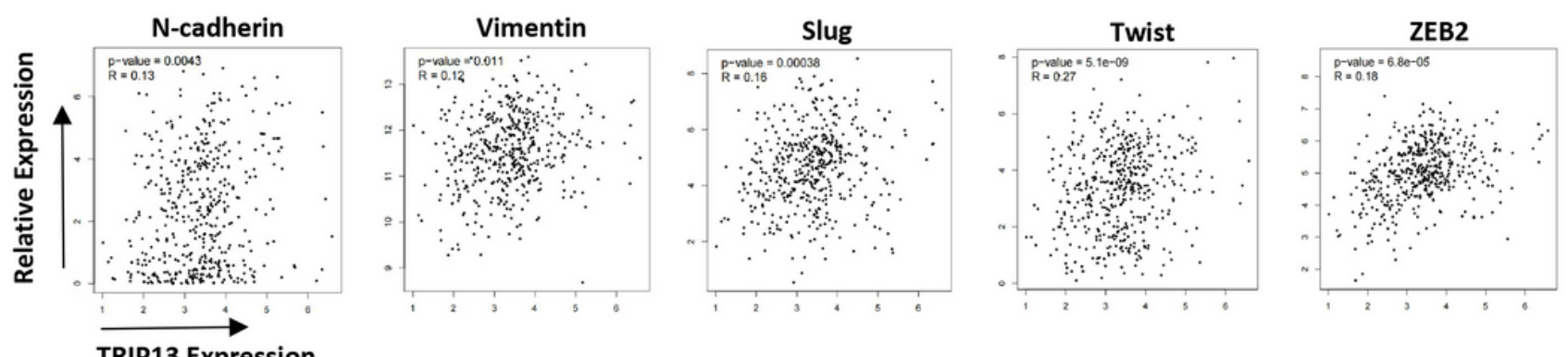

TRIP13 Expression

B

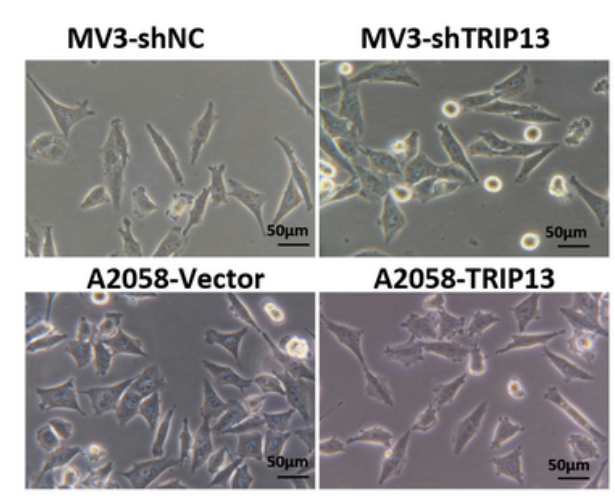

C

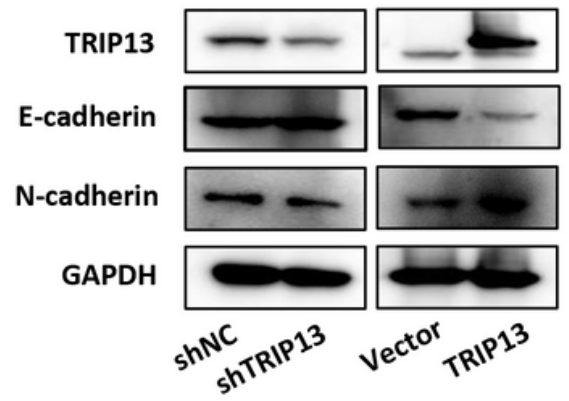

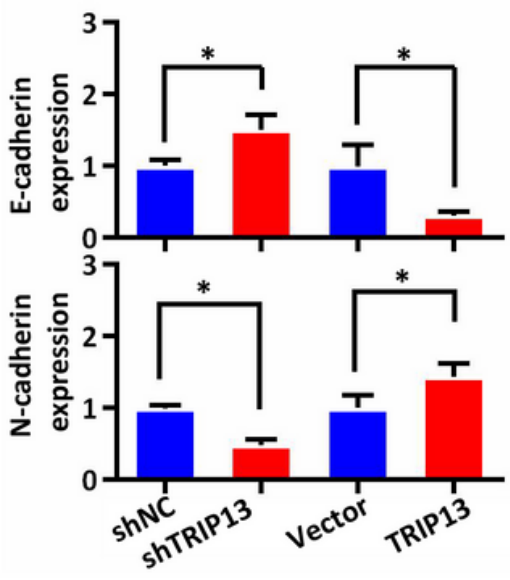

D

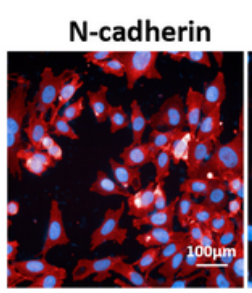

E-cadherin
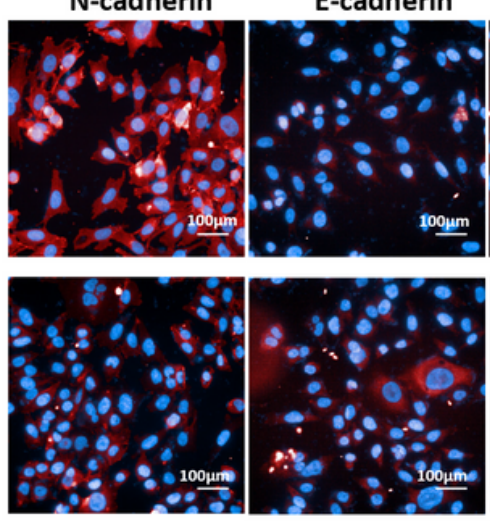
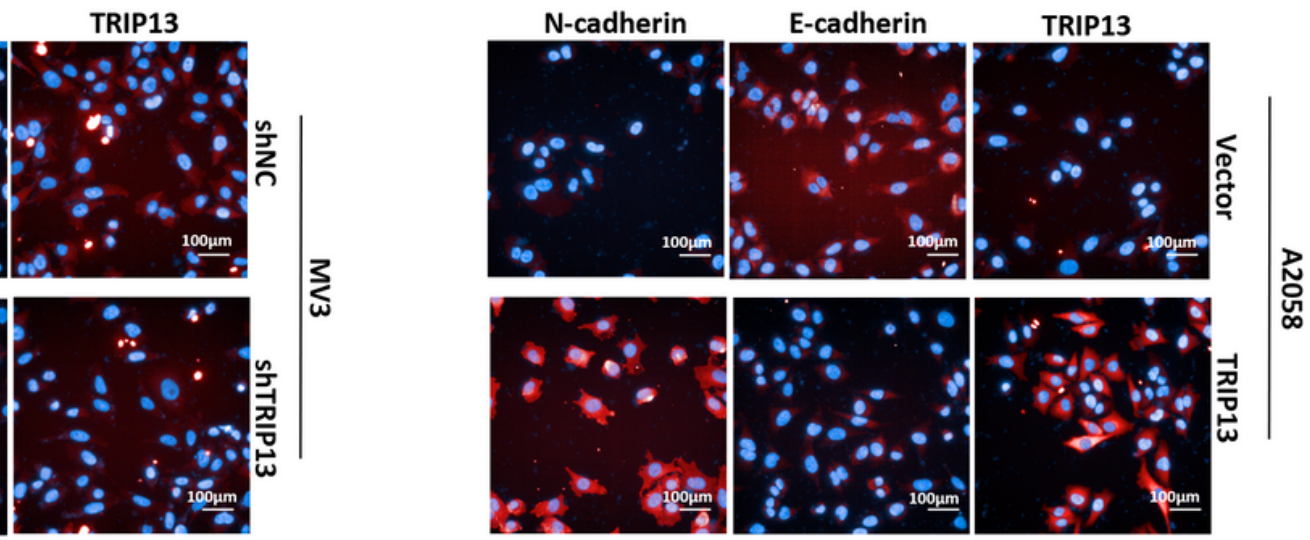

Figure 3 
TRIP13 induced EMT in melanoma cells. A. Relationship between N-cadherin, Vimentin, Slug, Twist, ZEB2 and TRIP13 in TCGA database in patients with melanoma. B. The shape of overexpressed / knockdown TRIP13 melanoma. C-D. Western blot analysis and Immunofluorescence of the expression of E-cadherin and N-cadherin in A2058-Vector/A2058-TRIP13/MV3-shNC/MV3-shTRIP13 cells. ${ }^{*} p<0.05$

A

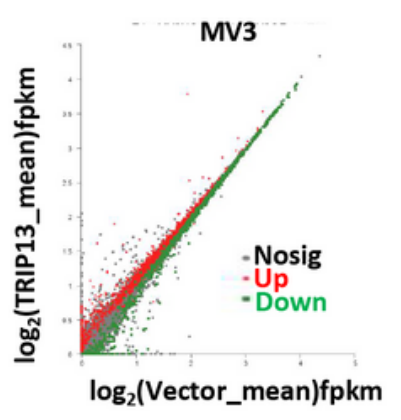

C

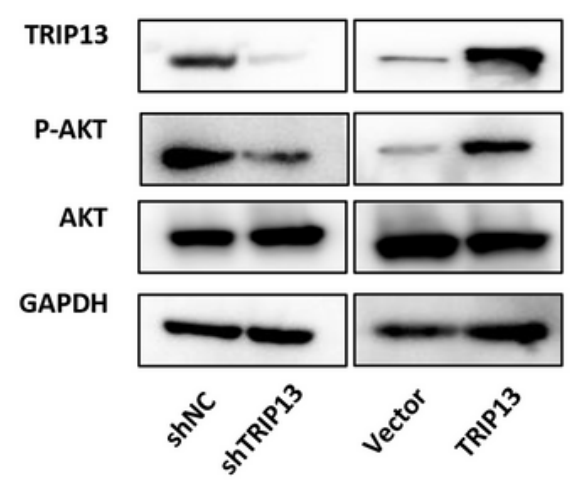

E

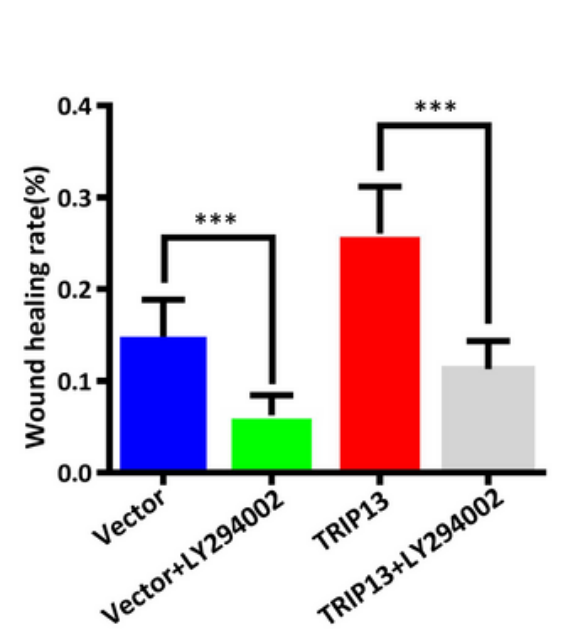

E
A2058

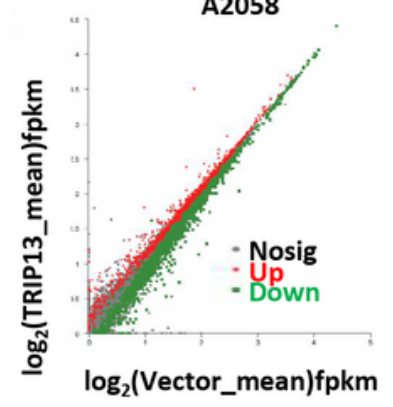

B

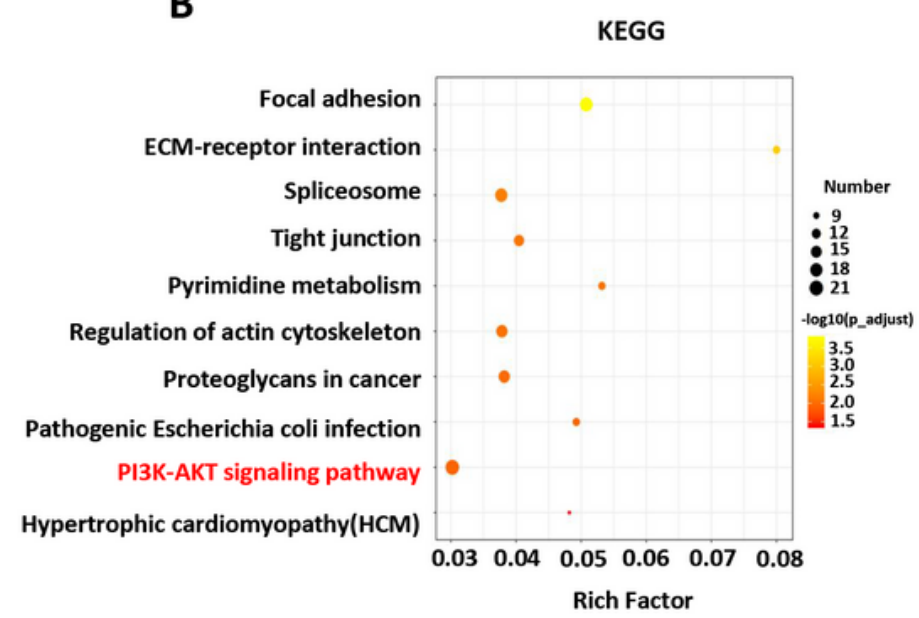

D

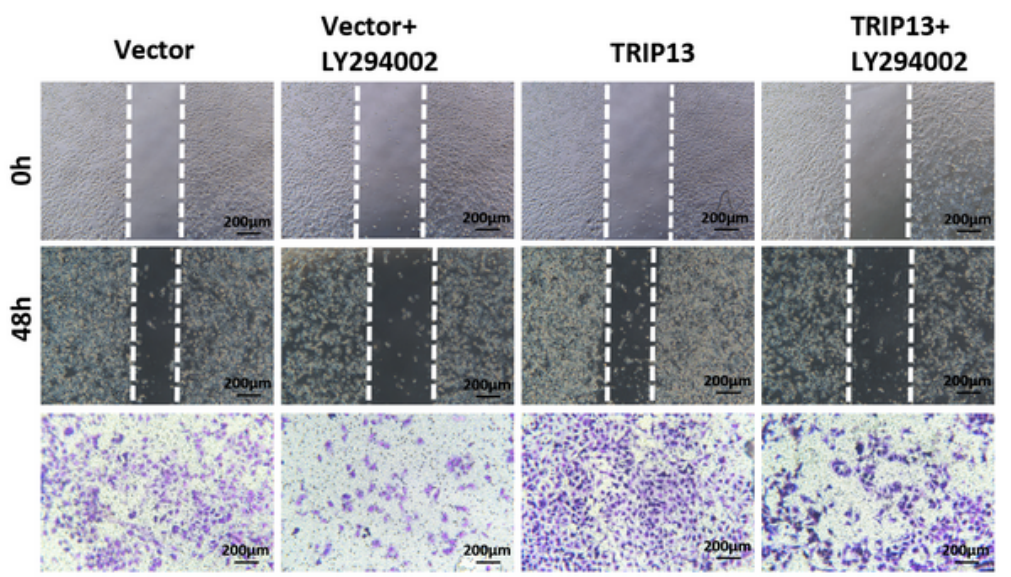

F

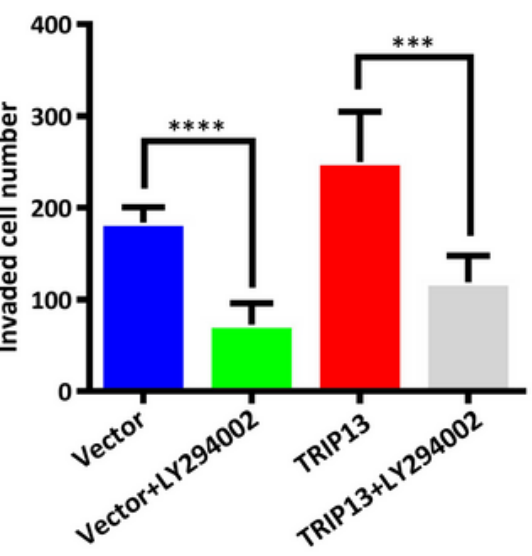

TRIP13
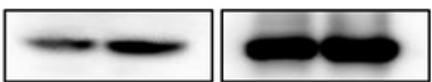

P-AKT
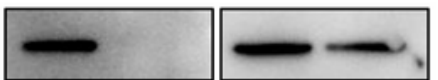

АKT
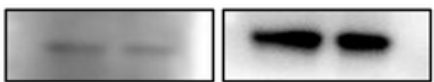

E-cadherin
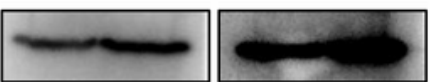

$\mathrm{N}$-cadherin
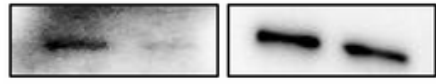

GAPDH
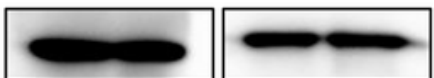

LY294002

Vector
TRIP13

Figure 4 
TRIP13 promotes melanoma migration, invasion and EMT via the PI3KJAKT pathway. A. DEGs in A2058shNC/A2058-TRIP13 and MV3-Vector/MV3-shTRIP13 cells were demonstrated by volcano plots. B. KEGG analyses of relative pathways were performed. C. The protein levels of TRIP13, p-AKT and AKT were detected in A2058-shNC, A2058-TRIP13, MV3-Vector and MV3-shTRIP13 by western blotting. D-E. Migration and invasion assays were performed in A2058-shNC, A2058-TRIP13 cells after incubation with LY294002. F. Western blotting was used to detect the levels of TRIP13, p-AKT, AKT, E-cadherin and Ncadherin in A2058-shNC and A2058-TRIP13cells after incubation with LY294002. ${ }^{* \star} p<0.001,{ }^{* \star \star *} p<$ 0.0001 
A

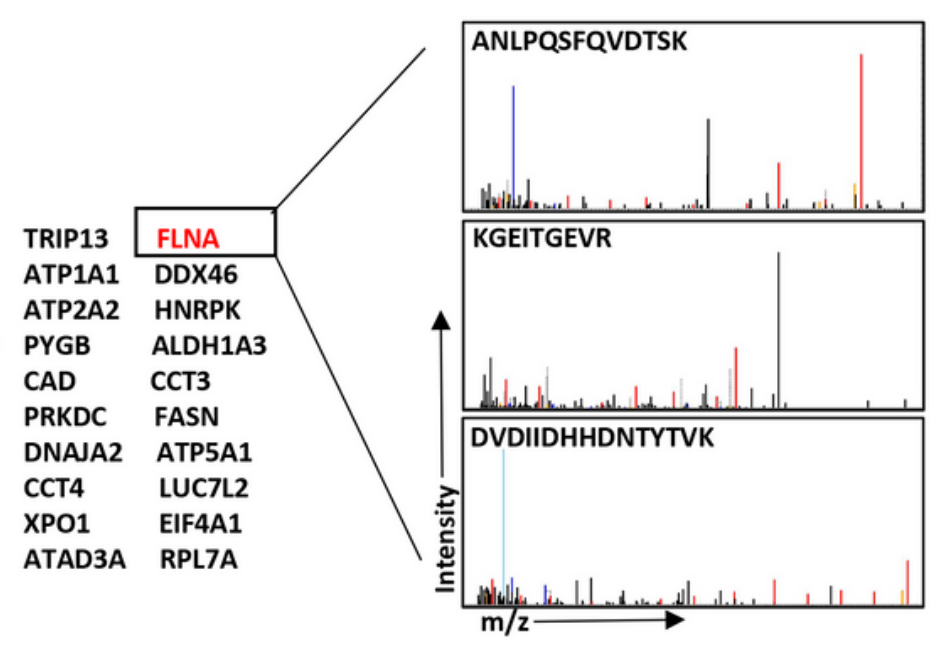

C
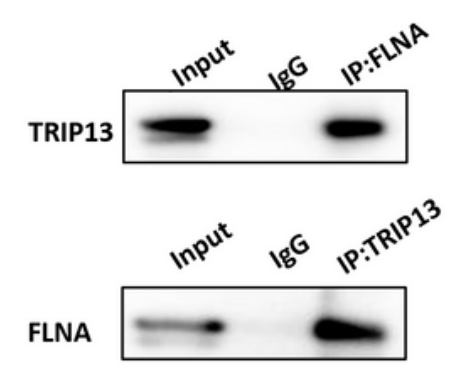

D
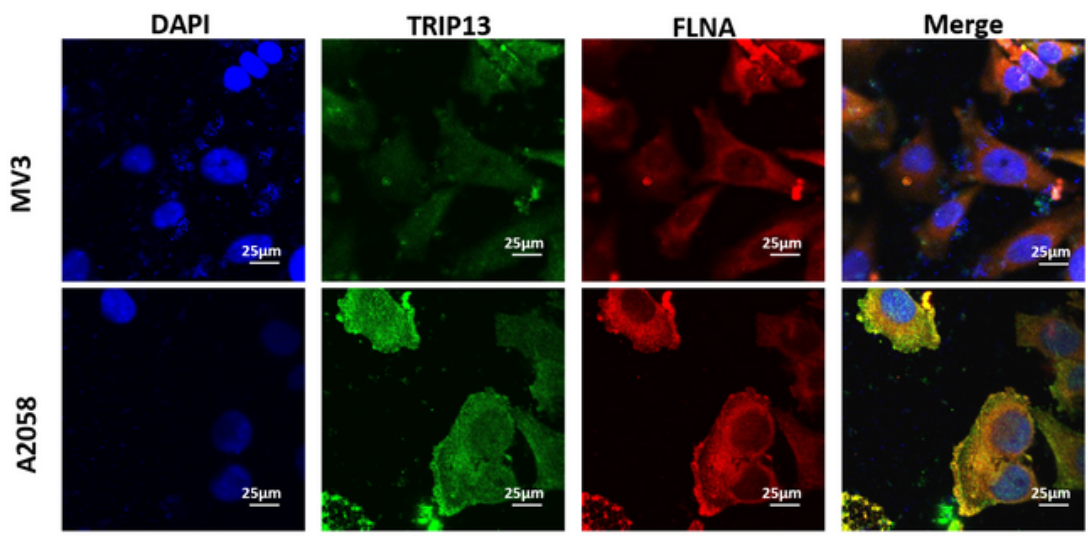

E
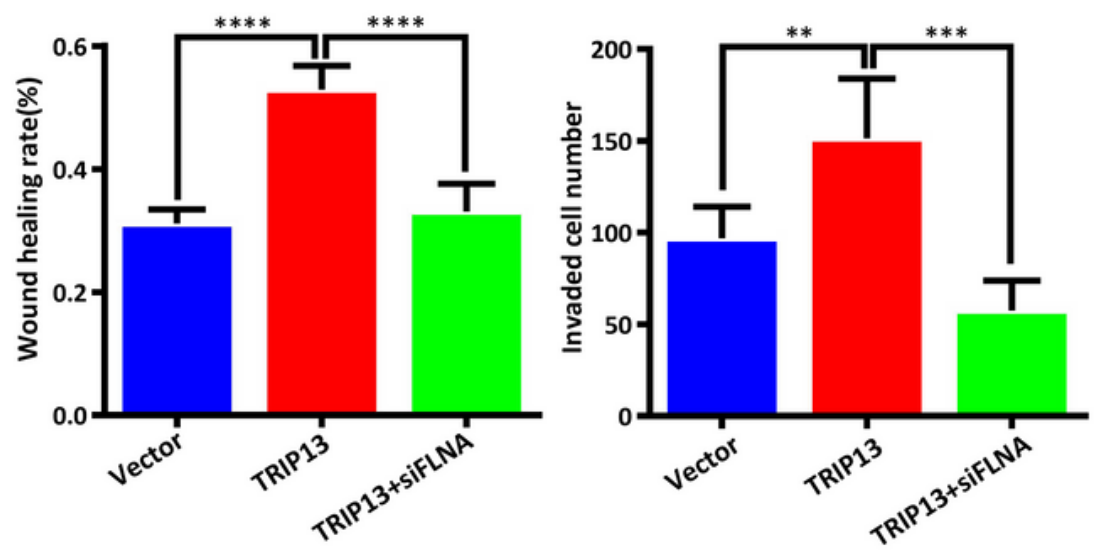

F

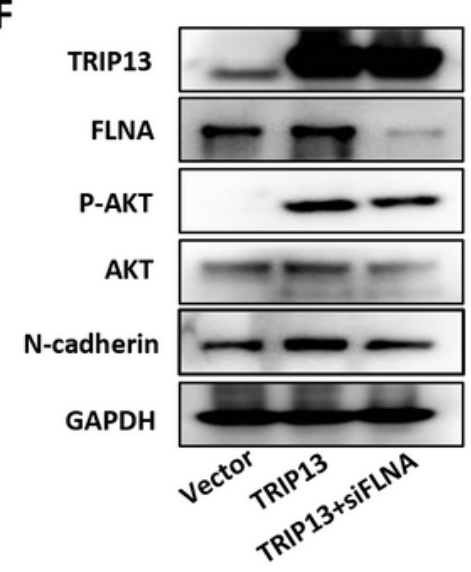

\section{Figure 5}

TRIP13 binds to FLNA and activates the PI3K/AKT pathway.A. Co-IP and MS detected the latent TRIP13combining molecule in A2058-Vector cells and A2058-TRIP13 cells. B. Co-IP was applied to confirm the interaction of TRIP13 and FLNA. C. Immunofluorescence was applied to confirm the colocalization of TRIP13 and FLNA. D. Western blot analysis of TRIP13 or FLNA expression in A2058 cells transfected with shTRIP13 or siFLNA. E. Cell migration and invasion were detected in related cells. F. Western blotting was 
used to detect the levels of TRIP13, FLNA, p-AKT, AKT, N-cadherin in related cells. ${ }^{*} p<0.05,{ }^{*} p<0.01$, ${ }^{* * *} p<0.001, * \star * * p<0.0001$

A

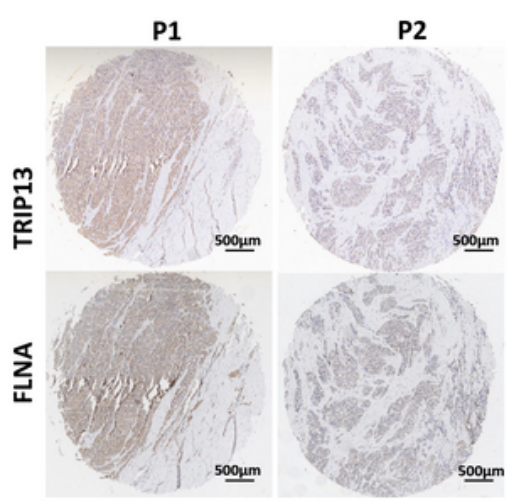

C

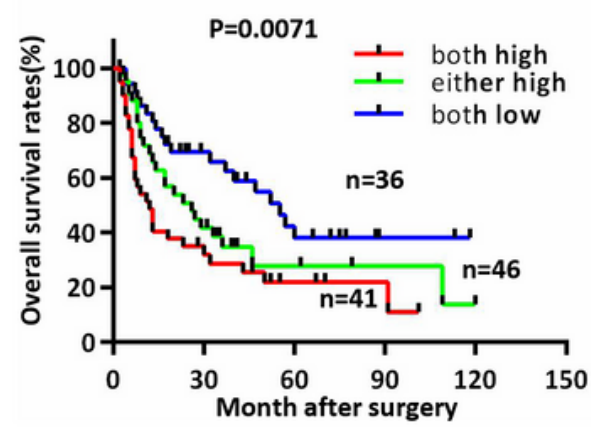

B

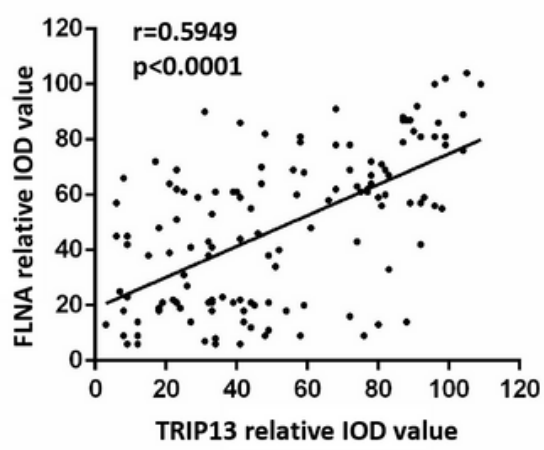

D

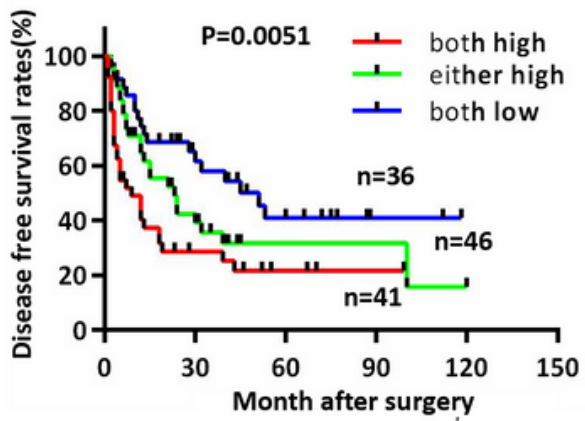

$\mathbf{E}$

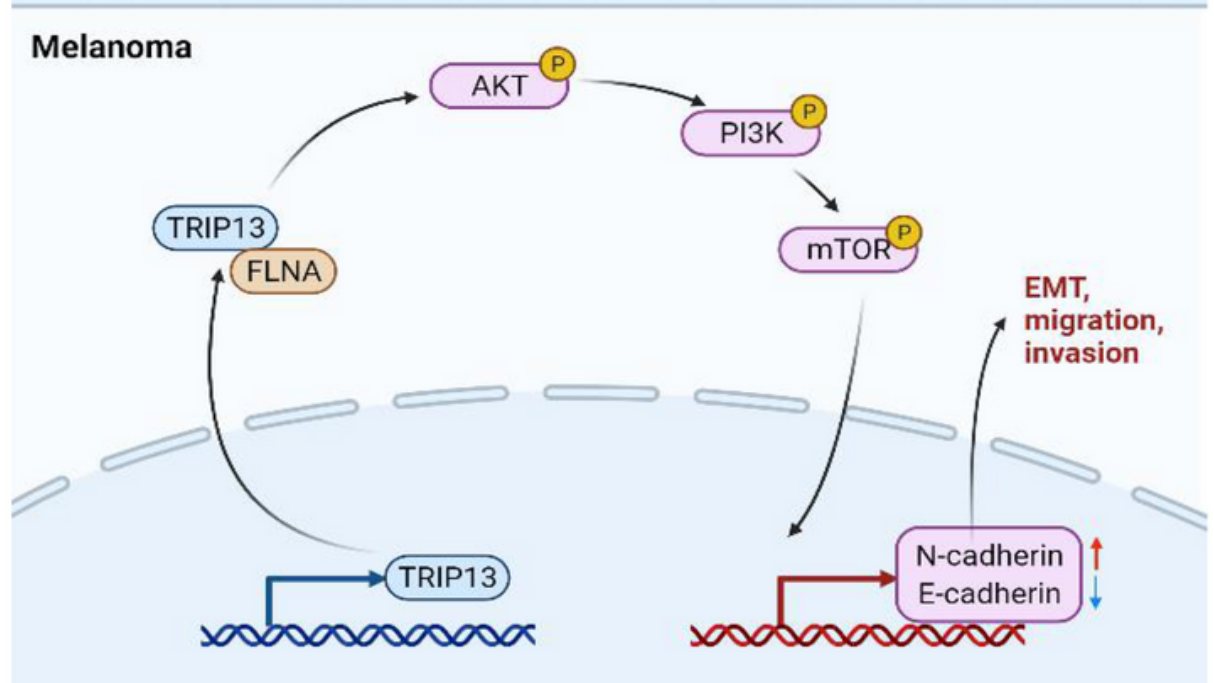

Figure 6

High expression of TRIP13 and FLNA indicated poor prognosis 
A. Immunohistochemistry of TRIP13 and FLNA in the TMA. B. Relationship between FLNA and TRIP13 in the TMA. C-D. Overall survival and disease free survival analyses of 123 melanoma patients according to TRIP13 and FLNA expression. E. Schematic diagram describing the biological function and mechanism of TRIP13 in melanoma progression. 